Article

\title{
Preparation of Theophylline-Benzoic Acid Cocrystal and On-Line Monitoring of Cocrystallization Process in Solution by Raman Spectroscopy
}

\author{
Yaohui Huang ${ }^{1}$, Ling Zhou ${ }^{1, *}$, Wenchao Yang ${ }^{1}$, Yang $\mathrm{Li}^{1}{ }^{1}$, Yongfan Yang ${ }^{1}$, Zaixiang Zhang ${ }^{1}$, \\ Chang Wang ${ }^{1}$, Xia Zhang ${ }^{1}$ and Qiuxiang Yin ${ }^{1,2,3, *}$ \\ 1 State Key Laboratory of Chemical Engineering, School of Chemical Engineering and Technology, Tianjin \\ University, Tianjin 300072, China \\ 2 Collaborative Innovation Center of Chemical Science and Engineering (Tianjin), Tianjin 300072, China \\ 3 Key Laboratory Modern Drug Delivery and High Efficiency in Tianjin University, Tianjin 300072, China \\ * Correspondence: zhouling@tju.edu.cn (L.Z.); qxyin@tju.edu.cn (Q.Y.)
}

Received: 5 June 2019; Accepted: 25 June 2019; Published: 27 June 2019

check for updates

\begin{abstract}
Pure theophylline-benzoic acid cocrystal was prepared via slurry and cooling crystallization in solution to overcome the disadvantages of existing preparation methods. The target cocrystal was characterized by powder X-ray diffraction (PXRD), thermalgravimetric analysis (TGA), differential scanning calorimetry (DSC) and Raman spectroscopy. The slurry and cooling cocrystallization process in solution was monitored via on-line Raman spectroscopy. The results obtained from on-line Raman monitoring can exhibit the transformation process from raw materials (theophylline and benzoic acid) to cocrystal and show the cocrystal formation rate. Comparing each transformation process under different conditions in slurry crystallization, we found that suspension density of raw materials and temperature both have an impact on the theophylline-benzoic acid cocrystal formation rate. It could be concluded that the cocrystal formation rate increased with the increase of suspension density of raw materials. Further under the same suspension density, higher temperature will accelerate theophylline-benzoic acid cocrystal formation. Meanwhile, various data from the cocrystallization process in cooling crystallization, including nucleation time, nucleation temperature and suitable cooling ending point can be gained from results of on-line Raman monitoring.
\end{abstract}

Keywords: cocrystal; solution crystallization; Raman spectroscopy; on-line monitoring; cocrystal formation

\section{Introduction}

Active pharmaceutical ingredients (API) can exist in different solid-state forms, such as polymorphs, salts, solvates, amorphous forms and cocrystals [1]. Cocrystal is a new class of pharmaceutical crystal form, which can improve the physicochemical properties of API effectively without affecting their internal structure [2]. Cocrystals are molecular complexes that contain two or more components together in the same crystal lattice, and their structures are based on hydrogen bonds, $\pi-\pi$ stacking, van der Waals forces and other non-covalent bonds [3]. In contrast to salts, cocrystal formation can be envisaged for acidic, basic and neutral APIs to open up new opportunities for the pharmaceutical industry [4]. Cocrystal synthesis has been carried out using a variety of methods, such as slow evaporation $[5,6]$, cooling crystallization [7,8], slurry crystallization [9], and mechanochemical synthesis and so on [10].

Among these synthesis methods, cooling and slurry crystallization are widely used in preparation of pharmaceutical cocrystals because they can achieve larger scale and higher yield of target cocrystals compared with other methods [7-9]. In recent studies, the research of pharmaceutical cocrystal is mainly focused on how to improve physicochemical properties of APIs and enhance their efficacy. 
Many articles have been published which are associated with the design, characterization analysis and properties of cocrystals [11-15]. However, the understanding of cocrystals is still far less than that of other solid forms and the understanding of the cocrystallization process in solution remains to be developed. With cooling and slurry crystallization, the cocrystal formation process is convenient to monitor and control by some on-line analysis methods, such as Raman spectroscopy, Fourier transform infrared spectroscopy (FTIR) and focused beam reflectance measurement (FBRM). Raman spectroscopy, as a kind of reliable and effective technique that can detect both solid and liquid phases, is more suitable to monitor cocrystal formation [16-19].

Theophylline (TP) is a drug for asthma therapy and chronic obstructive pulmonary disease treatment [20]. However, in clinical application, the low water solubility limits its bioavailability. For this reason, new molecular compounds containing theophylline need to be developed to expand its clinical application. Therefore, the synthesis of cocrystal is research focused on improving physicochemical properties of theophylline [21-26]. Theophylline has great potential to form cocrystal due to the carbonyl groups and aromatic nitrogen atoms in the structure, which are readily to form hydrogen bonds. Hence, theophylline is a good model molecule to research cocrystal formation and investigate the cocrystallization mechanism. According to the literature, the cocrystals of theophylline have been successfully prepared with several carboxylic acids, such as glutaric acid, oxalic acid, and benzoic acid $[10,27,28]$. Benzoic acid (BA) occurs naturally in many plants and serves as an intermediate in the biosynthesis of many secondary metabolites [29]. Benzoic acid is a good non-toxic cocrystal coformer because of the carboxyl group in its structure, which can form many kinds of cocrystals with different APIs. The 1:1 theophylline-benzoic acid cocrystal has been reported to be prepared via neat grinding and slow evaporation [27,30]. These two methods are mostly commonly used in cocrystal screening, however are not conducive for robust scaling because of the inherent limitations of the techniques and solution crystallization most widely used to achieve large-scale production of crystals $[13,31]$. Therefore, solution crystallization need to be explored to prepare pure theophylline-benzoic acid cocrystal.

Our research focuses on preparation and on-line monitoring of the formation process of theophylline-benzoic acid cocrystal. The molecule structure of TP-BA cocrystal at a stoichiometric molar ratio of 1:1 from the Cambridge Crystallographic Data Centre is shown in Figure 1 [27]. In this study, we successfully prepared pure TP-BA cocrystal via solution crystallization (slurry and cooling crystallization) and characterized the solid phase of the theophylline-benzoic acid cocrystal by different analysis methods. In our research, we investigated the TP-BA cocrystal formation process in slurry and cooling crystallization by on-line Raman spectroscopy. Furthermore, the influence of suspension density of raw materials (theophylline and benzoic acid) and temperature on cocrystal formation rate in slurry crystallization was demonstrated. Further, the nucleation temperature and suitable cooling ending point in cooling crystallization can be also achieved by on-line Raman monitoring.

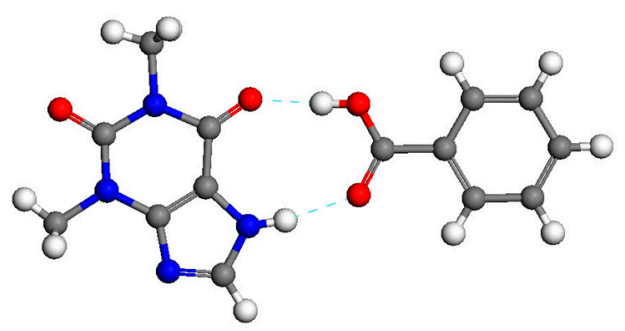

Figure 1. The molecules of theophylline-benzoic acid (TP-BA) cocrystal at a stoichiometric molar ratio of 1:1 (grey atoms: C; red atoms: O; blue atoms: $\mathrm{N}$; white atoms: $\mathrm{H}$ ). 


\section{Materials and Methods}

\subsection{Materials}

Theophylline (Form II) was purchased from Aladdin-Reagent Technology Co. Ltd. (Shanghai, China) and benzoic acid was purchased from Tianjin Guangfu Chemical Reagent Co. (Tianjin, China). Deionized water and analytic grade methanol (Tianjin Kewei Chemical Reagent Co., Tianjin, China) were used in this work. All materials were used without further purification. Table 1 shows mass fraction purity and provenance of the materials.

Table 1. Description of materials used in this paper.

\begin{tabular}{cccc}
\hline Chemical & Source & Mass Fraction Purity & Purification Method \\
\hline Theophylline & $\begin{array}{c}\text { Aladdin-Reagent Technology Co. Ltd. } \\
\text { (Shanghai, China) }\end{array}$ & $>0.990$ & GCa \\
Benzoic acid & $\begin{array}{c}\text { Tianjin Guangfu Chemical Reagent Co. } \\
\text { (Tianjin, China) }\end{array}$ & $>0.990$ & GCa \\
Deionized water & $\begin{array}{c}\text { Tianjin Kewei Chemical Co. Ltd. } \\
\text { (Tianjin, China) } \\
\text { Methanol }\end{array}$ & $\begin{array}{c}\text { Tianjin Kewei Chemical Co. Ltd. } \\
\text { (Tianjin, China) }\end{array}$ & $>0.995$ \\
\hline
\end{tabular}

GCa: Gas-liquid chromatography.

\subsection{Preparation of Theophylline-Benzoic Acid Cocrystal}

Theophylline-benzoic acid cocrystal was synthesized by slurry and cooling crystallization in methanol/water mixture $(\mathrm{V}: \mathrm{V}=1: 5)$. In our pre-experiments, the target cocrystal was synthesized in pure acetonitrile and the ratio of API and coformer used for preparation was 1:5 due to the solubility difference between theophylline and benzoic acid, which resulted in benzoic acid being wasted. The mixed solvents (methanol/water mixture) decreased the solubility difference between TP and BA and modified the raw materials ratio to 1:1 to avoid wasting of coformer.

In the slurry crystallization, TP-BA cocrystal solubility in methanol/water mixture solution $(\mathrm{V}: \mathrm{V}=1: 5)$ was determined by static method with high-performance liquid chromatography (HPLC), which was $0.087 \mathrm{~mol} / \mathrm{L}$ at $298.15 \mathrm{~K}$. A total of $5.95 \mathrm{mmol} \mathrm{TP}$ and $5.95 \mathrm{mmol} \mathrm{BA}$ was added a $36 \mathrm{~mL}$ methanol/water mixture to make a suspension of both TP and BA. Due to the total added materials and cocrystal solubility, the suspension density was $0.078 \mathrm{~mol} / \mathrm{L} \mathrm{TP}$ and $0.078 \mathrm{~mol} / \mathrm{L} \mathrm{BA}$ at $298.15 \mathrm{~K}$. The TP and BA suspension was stirred magnetically in a water bath at $298.15 \mathrm{~K}$ for at least $5 \mathrm{~h}$ to reach equilibrium. Then the cocrystal product was filtered from the suspension and dried at $313.15 \mathrm{~K} \mathrm{for} 12 \mathrm{~h}$. In the cooling crystallization, $3.5 \mathrm{mmol} \mathrm{TP}$ and $3.5 \mathrm{mmol} \mathrm{BA}$ were dissolved in $36 \mathrm{ml}$ methanol/water mixture $(\mathrm{V}: \mathrm{V}=1: 5)$. The solution temperature was kept at $313.15 \mathrm{~K}$ for $1 \mathrm{~h}$, and then cooled to $278.15 \mathrm{~K}$ with a $9 \mathrm{~K} / \mathrm{h}$ cooling rate. The TP-BA cocrystal can be formed in the cooling process. The solid product of cooling crystallization was isolated over a filter paper (Whatman $2.5 \mu \mathrm{m}$ grade, Shanghai, China) using vacuum filtration and dried for $12 \mathrm{~h}$ at $313.15 \mathrm{~K}$ in an oven. The temperature of the water bath was controlled by a thermostat (XODC-2006, Xianou Laboratory Instrument Works Co., Ltd., Nanjing, China), and the system temperature variation for all the measurements was found to be less than $\pm 0.1 \mathrm{~K}$. The cocrystal products gained from slurry and cooling crystallization were analyzed by powder X-ray diffraction (PXRD, Rigaku, Tokyo, Japan) and high-performance liquid chromatography (HPLC, Agilent Technologies, Inc., Carpinteria, CA, USA) to determine solid phase composition. Then TP-BA cocrystal was characterized by differential scanning calorimetry (DSC, Mettler Toledo, Greifensee, Switzerland), thermogravimetric analysis (TGA, Mettler Toledo, Greifensee, Switzerland) and Raman spectroscopy (Kaiser Raman RXN2, Ann Arbor, MI, USA). Raman spectroscopy was used to monitor the cocrystal formation process during slurry and cooling crystallization. 


\subsection{Monitoring Cocrystallization Process of Theophylline and Benzoic Acid in Slurry Crystallization}

To explore the factors affecting the TP-BA cocrystal formation process in slurry crystallization, we performed four sets of slurry experiments in a methanol/water mixture $(\mathrm{V}: \mathrm{V}=1: 5)$. The corresponding temperature, initial concentration and suspension density of raw materials are listed in Table 3. The suspension density was calculated from the total concentration and cocrystal solubility at $298.15 \mathrm{~K}$ and $313.15 \mathrm{~K}$. Cocrystal solubility at $298.15 \mathrm{~K}$ and $313.15 \mathrm{~K}$ was determined by static method with HPLC. The ratio of TP and BA in cocrystal products was analyzed using HPLC to confirm TP-BA cocrystal purity. This cocrystallization process was monitored by Raman spectroscopy for $5 \mathrm{~h}$ to reach the equilibrium. The whole spectra was obtained at a spectral range of $200-1800 \mathrm{~cm}^{-1}$.

\subsection{Analytical Methods}

A Raman spectrometer (RXN2, Kaiser Optical systems, Inc., Ann Arbor, MI, USA) was used for both off-line measurement of solid samples and on-line monitoring of cocrystal formation process in slurry and cooling crystallization. In the Raman spectroscopy, the spectral resolution and the excitation wavelength of the laser were $5 \mathrm{~cm}^{-1}$ and $785 \mathrm{~nm}$, respectively.

The PXRD patterns of the TP-BA cocrystal were obtained using a powder diffractometer (D/MAX 2500, Rigaku, Tokyo, Japan) with a Cu K $\alpha$ radiation (1.54 $\AA$ ), tube voltage of $40 \mathrm{kV}$, and current of $100 \mathrm{~mA}$. Data were collected between $2^{\circ}$ and $40^{\circ}$ in $2 \theta$ with steps of $0.05^{\circ}$ and a dwelling time of $1 \mathrm{~s}$ per step.

Thermal measurements were performed by TGA/DSC (1/500, Mettler Toledo, Greifensee, Switzerland) protected by nitrogen atmosphere. Experimental conditions were followed as pans of $40 \mu \mathrm{L}$ volume with a heating rate of $10 \mathrm{~K} / \mathrm{min}$ and a scan range from $298.15 \mathrm{~K}$ to $573.15 \mathrm{~K}$.

The ratio of TP and BA in cocrystal products was analyzed by high-performance liquid chromatography (HPLC). The HPLC is equipped with a UV-vis spectrophotometer detector and uses a C18 column (Extend, $5 \mu \mathrm{m}, 4.6 \times 250 \mathrm{~mm}$, Agilent Technologies, Inc., Carpinteria, CA, USA) to separate TP and BA. The mobile phase was composed of $60 \%$ methanol and $40 \%$ water with $0.1 \%$ trifluoroacetic acid. The flow was set at $1 \mathrm{~mL} / \mathrm{min}$ and the sample injection volume was $20 \mu \mathrm{L}$. Absorbance was monitored at $250 \mathrm{~nm}$. Data collection and processing were performed using software from Agilent Technologies, Inc., Carpinteria, CA, USA.

\section{Results and Discussion}

\subsection{Solid Phases Characterization of Theophylline-benzoic Acid Cocrystal}

The powder X-ray diffraction patterns of TP, BA, physical mixture, solid products obtained in slurry and cooling crystallization, and the calculated data of TP-BA cocrystal from single crystal X-ray data [27] are presented in Figure 2a. The PXRD patterns of the cocrystal products obtained in slurry and cooling crystallization are significantly different from those of TP, BA and physical mixture, but the same as the PXRD pattern calculated by single-crystal X-ray data [27]. In detail, the peaks at $6.10^{\circ}$ and $17.58^{\circ}$, which are characteristics peaks of TP, are absent in the PXRD patterns of the cocrystal, as is the characteristic peak of BA at $7.98^{\circ}$. Meanwhile, some new peaks appear at $11.40^{\circ}$ and $19.46^{\circ}$ in the cocrystal product pattern, which are the same as the PXRD pattern of the calculated data of the TP-BA cocrystal. Therefore, the formation of TP-BA cocrystal can be confirmed by the changes in the PXRD patterns. The ratio of TP and BA in cocrystal products is obtained as 1:1 by HPLC. 


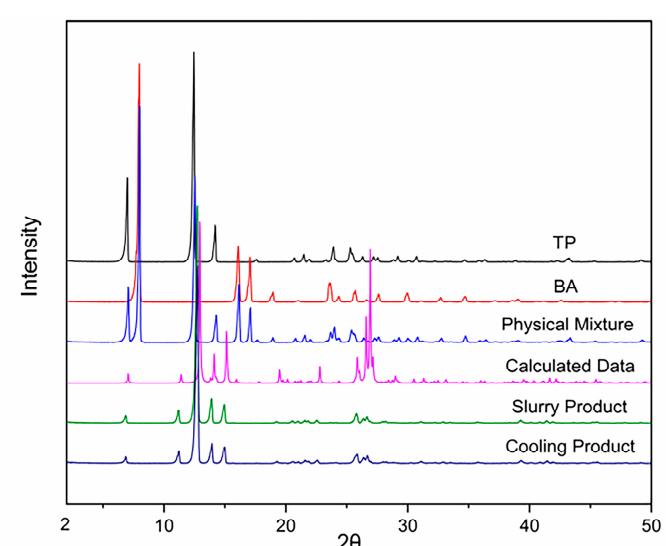

(a)

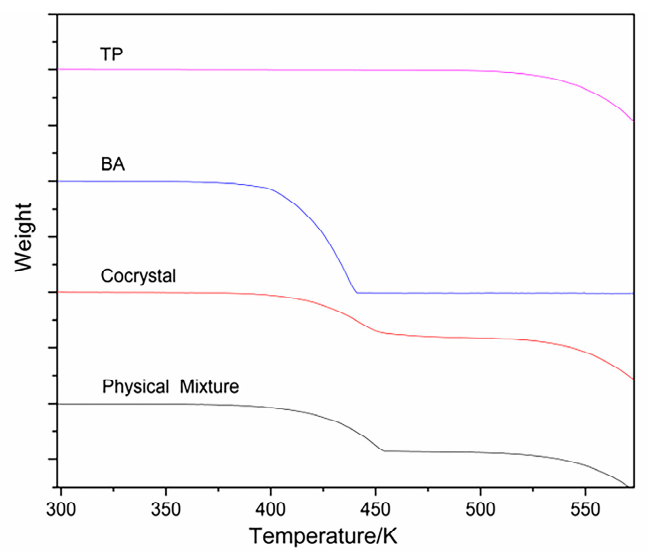

(c)

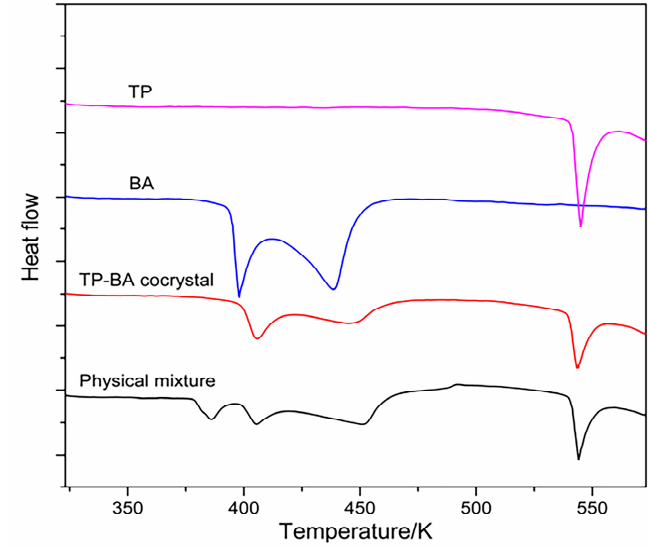

(b)

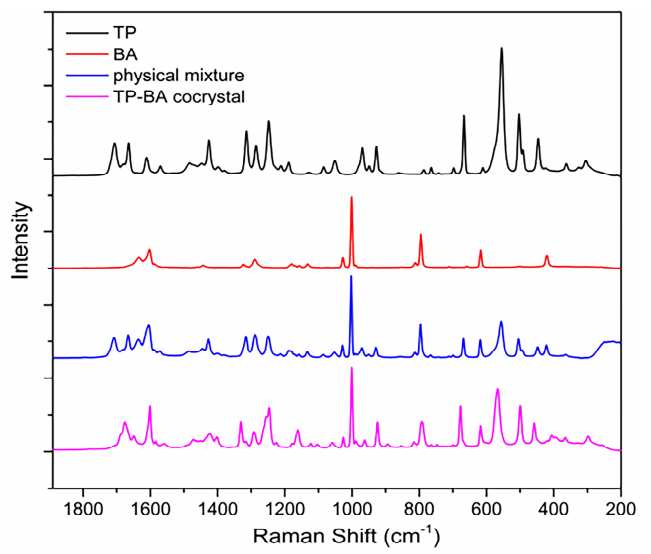

(d)

Figure 2. The solid characterization spectra of TP, BA, physical mixture and TP-BA cocrystal: (a) powder X-ray diffraction (PXRD); (b) differential scanning calorimetry (DSC); (c) thermogravimetric analysis (TGA); (d) Raman spectroscopy.

The DSC and TGA curves of TP, BA, TP-BA cocrystal and physical mixture are shown in Figure 2. The melting points of theophylline and benzoic acid are $545.5 \mathrm{~K}$ and $395.1 \mathrm{~K}$, respectively. In the DSC curve of the TP-BA cocrystal, the first endothermic peak at around $411.1 \mathrm{~K}$ is the melting point of cocrystal, which is significantly different from API and coformer. The second peak indicates the cocrystal decomposing at $455.3 \mathrm{~K}$. Around this temperature, benzoic acid breaks away from the structure of the TP-BA cocrystal, and theophylline recrystallizes to the solid phase. In the TGA curve of cocrystal, at $455.3 \mathrm{~K}$ solid weight begins decreasing and the total weightlessness is about $40 \%$, which is equal to the mass fraction of BA in cocrystal. The last peak in DSC curve of the TP-BA cocrystal at $545.5 \mathrm{~K}$ is the melting point of the remaining theophylline. Moreover, the DSC curve of the physical mixture has four endothermic peaks. The peak at $384.5 \mathrm{~K}$ indicates that solid theophylline and solid benzoic acid form the TP-BA cocrystal at this temperature and the next peak at $411.1 \mathrm{~K}$ is the melting point of the cocrystal, which corresponds with the curve of pure cocrystal. Further, the peaks at $455.3 \mathrm{~K}$ and $545.5 \mathrm{~K}$ represent the decomposition of BA from the cocrystal and the melting point of the remaining TP, respectively, which are as same as those in the DSC curve of cocrystal.

Figure $2 \mathrm{~d}$ shows the Raman spectra of the target cocrystal, physical mixture, TP and BA. It is obviously that the spectra of the cocrystal is different from those of TP and BA. For instance, TP and BA have the characteristic peaks at 1688, 1323, 1171 and $918 \mathrm{~cm}^{-1}$, while the characteristic peaks of the cocrystal are at $1678,1331,1161$ and $925 \mathrm{~cm}^{-1}$. The difference between the Raman spectra of the cocrystal and raw materials can be used to identify TP-BA cocrystal formation and monitor the cocrystallization process in solution. 


\subsection{Crystal Structure Analysis of TP-BA Cocrystal}

The crystallographic data of the TP-BA cocrystal is obtained from Cambridge Crystallographic Data Centre (CCDC) [27] and the crystal structure is shown in Figure 3. Each TP molecule is connected to one BA molecule via two hydrogen bonds to form a dimer. One hydrogen bond is formed between the acidic nitrogen atom on the imidazole ring from the TP molecule and the carboxyl oxygen atom from the BA molecule. The other is formed between the carbonyl group from the TP molecule and the carboxyl group of the BA molecule. The dimers of TP-BA molecules are arranged parallel to the b-c plane, then form stacks along the a-axis. The space group of the TP-BA cocrystal is monoclinic, $\mathrm{P} 2{ }_{1} / n$ and has cell parameters $a=6.98690$ (17) $\AA, b=25.10944$ (84) $\AA, c=8.60685$ (30) $\AA, \beta=108.5597$ (18), and $\mathrm{V}=1431.431(78) \AA^{3}$. From the TP-BA cocrystal structure, two hydrogen bonds' interaction results in changes in the stretching vibrations of the bonds in TP and BA molecules' structures, which can cause peak shifts in the Raman spectra (Figure 4). For example, the peak at $1688 \mathrm{~cm}^{-1}$ is observed in both Raman spectra of TP and the physical mixture, while there is no peak at a similar position in the Raman spectra of BA. It indicates that this peak should be caused by stretching vibrations of the $\mathrm{N}-\mathrm{C}=\mathrm{O}$ bond in TP molecule. The hydrogen bond, $\mathrm{N}-\mathrm{C}=\mathrm{O} \cdot \mathrm{H}-\mathrm{O}$, formation, causes this peak to shift from $1688 \mathrm{~cm}^{-1}$ to $1678 \mathrm{~cm}^{-1}$. Similarly, in the spectra of TP and physical mixture we can see a peak at $918 \mathrm{~cm}^{-1}$, which represents N-H on the imidazole ring. After forming a hydrogen bond, $\mathrm{N}-\mathrm{H} \cdot \mathrm{O}=\mathrm{C}$, this peak shifts to $925 \mathrm{~cm}^{-1}$. Therefore, during the cocrystallization process, the Raman spectra of the solution would change resulting from hydrogen bonds' interaction between TP and BA molecules, which can be used to monitor the TP-BA cocrystal formation process.

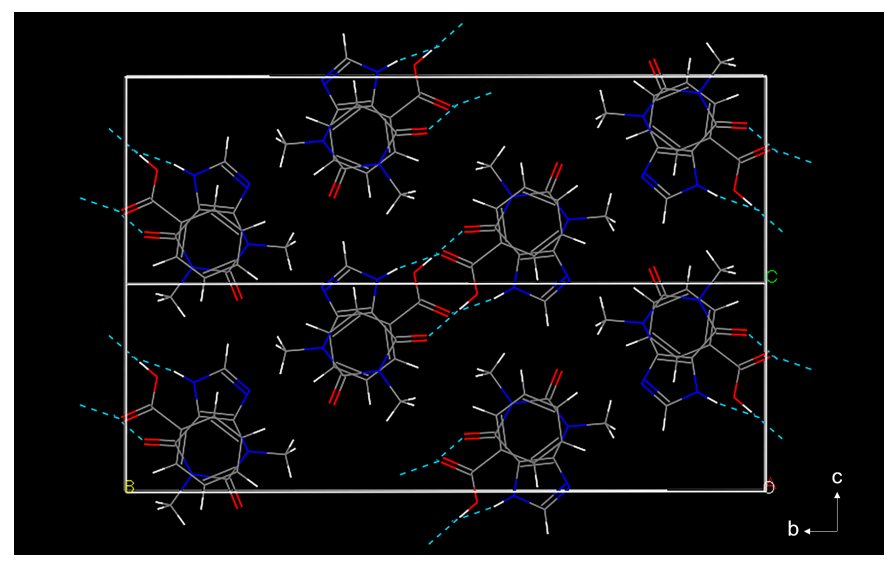

Figure 3. The crystal structure of the TP-BA cocrystal (grey color: C; red color: O; blue color: N; white color: $\mathrm{H})$.

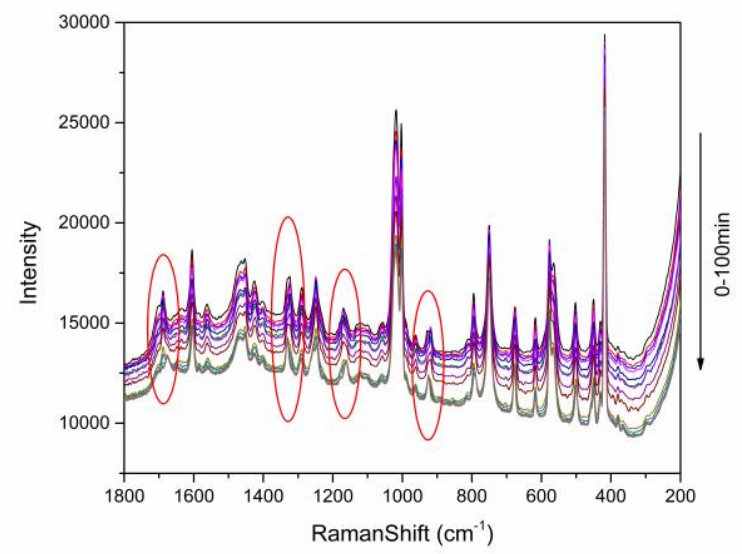

Figure 4. The variation of the Raman spectra of TP-BA cocrystal formation in slurry crystallization. The time intervals of the Raman spectra are listed in Table 2. 
Table 2. The time intervals of the Raman spectra shown in Figure 4.

\begin{tabular}{cccc}
\hline Total Spectra Number & Total Recording Time/min & Time Point of First Spectra/min & Time Interval/min \\
\hline 20 & 100 & 5 & 5 \\
\hline
\end{tabular}

\subsection{On-Line Monitoring of TP-BA Cocrystal Formation Process in Slurry Crystallization}

The Raman spectra at $200-1800 \mathrm{~cm}^{-1}$ obtained from slurry crystallization at $298.15 \mathrm{~K}$ is shown in Figure 4. In this figure, the shifts of the Raman spectra within operating time can be observed. The main shifts ranges are at (1) $1678-1688 \mathrm{~cm}^{-1}$, (2) $1323-1331 \mathrm{~cm}^{-1}$, (3) $1161-1171 \mathrm{~cm}^{-1}$, and (4) $918-925 \mathrm{~cm}^{-1}$, respectively, and are marked with circles in Figure 4, which are corresponding with the off-line Raman spectra of the solid phase of TP, BA and cocrystal. That demonstrates that on-line Raman spectroscopy can be used to monitor the TP-BA cocrystal formation process in slurry crystallization.

Figure 5a presents the Raman spectra of the range of $1640-1720 \mathrm{~cm}^{-1}$ obtained from slurry crystallization at $298.15 \mathrm{~K}$. A main peak shift from $1688 \mathrm{~cm}^{-1}$ to $1678 \mathrm{~cm}^{-1}$ can be observed in the spectra. The solid-state Raman spectra of TP, BA and cocrystal were analyzed in Section 3.1. The characteristic peak at $1688 \mathrm{~cm}^{-1}$ can represent raw materials in the suspension, while the peak at the $1678 \mathrm{~cm}^{-1}$ could represent the TP-BA cocrystal existing in the slurry. Further, the peak intensity in the Raman spectra is positively related with the concentration of the compounds. The appearance of the characteristic peak, which represents the cocrystal, could demonstrate TP-BA cocrystal nucleation, and the decrease of the characteristic peak intensity of drug and coformer indicates raw material consumption, which is caused by the transformation from raw materials to cocrystal. Therefore, the target cocrystal formation can be identified by monitoring the characteristic peak intensity of the TP-BA cocrystal and raw materials. At the beginning of slurry crystallization only the peak at $1688 \mathrm{~cm}^{-1}$ could be seen from the spectra. As the cocrystallization process proceeds, the peak at $1678 \mathrm{~cm}^{-1}$ appears and the intensity starts to increase, which proves the TP-BA cocrystal formation. The intensity at $1630 \mathrm{~cm}^{-1}$ as the baseline is used to compare the intensity of two peaks, 1678 and $1688 \mathrm{~cm}^{-1}$. The difference between the intensity of characteristics peaks and the baseline is named the "relative peak intensity". The changes of the relative peak intensity of $1688 \mathrm{~cm}^{-1}$ and $1678 \mathrm{~cm}^{-1}$ are shown in Figure $5 \mathrm{~b}$. We can see that the relative intensity of $1678 \mathrm{~cm}^{-1}$ increases from -250 to 250 over $44 \mathrm{~min}$ and then maintains a stable level. Meanwhile, the relative peak intensity of $1688 \mathrm{~cm}^{-1}$ decreases from 1500 to 240 during the same period. The results indicate that at this condition the transformation from raw materials to cocrystal finishes at $44 \mathrm{~min}$, and can help us to improve the design of cocrystallization experiments and save more resources and time.

The spectra of $1270-1360 \mathrm{~cm}^{-1}$ can be observed in Supplementary Materials Figure S1a. During the cocrystallization process, the intensity of the peak at $1323 \mathrm{~cm}^{-1}$ keeps decreasing, while the intensity of the peak at $1331 \mathrm{~cm}^{-1}$ gradually increases within the operating time. We choose the intensity of $1306 \mathrm{~cm}^{-1}$ as the baseline for the characteristic peaks of 1323 and $1331 \mathrm{~cm}^{-1}$. The relative intensity of these two peaks is calculated by the above method and presented in Supplementary Figure S1b. The relative peak intensity of $1323 \mathrm{~cm}^{-1}$ decreases from 2000 to 500 , while that of the peak at $1331 \mathrm{~cm}^{-1}$ rises from 1200 to 1750 over a period of about $44 \mathrm{~min}$. Further, the time of transformation from raw materials to cocrystal obtained from the relative intensity change is the same as for the above analysis.

Moreover, two peak shifts can be seen at the range of $1120-1200 \mathrm{~cm}^{-1}$ and $890-940 \mathrm{~cm}^{-1}$, respectively. One peak at $1171 \mathrm{~cm}^{-1}$ shifts to $1161 \mathrm{~cm}^{-1}$ (Supplementary Figure S2a) and another moves from 918 to $925 \mathrm{~cm}^{-1}$ (Supplementary Figure S3a). When the slurry crystallization begins, we can only see the peak at 1171 and $918 \mathrm{~cm}^{-1}$. As the cocrystal formation proceeds, the peaks at 1161 and $925 \mathrm{~cm}^{-1}$ appear. The intensities of 1140 and $900 \mathrm{~cm}^{-1}$ are used as the baseline. In the Supplementary Figure S2b, the relative intensity of the peak at $1171 \mathrm{~cm}^{-1}$ reduces from 1300 to 350 and the relative peak intensity at $1161 \mathrm{~cm}^{-1}$ increases from 620 to 1050 over a period of $44 \mathrm{~min}$. Similarly, the relative intensity of the peak at $918 \mathrm{~cm}^{-1}$ reduces from 1400 to 300 , however the intensity of the peak at $925 \mathrm{~cm}^{-1}$ increases from 700 to 1250 at the same time (Supplementary Figure S3b). 


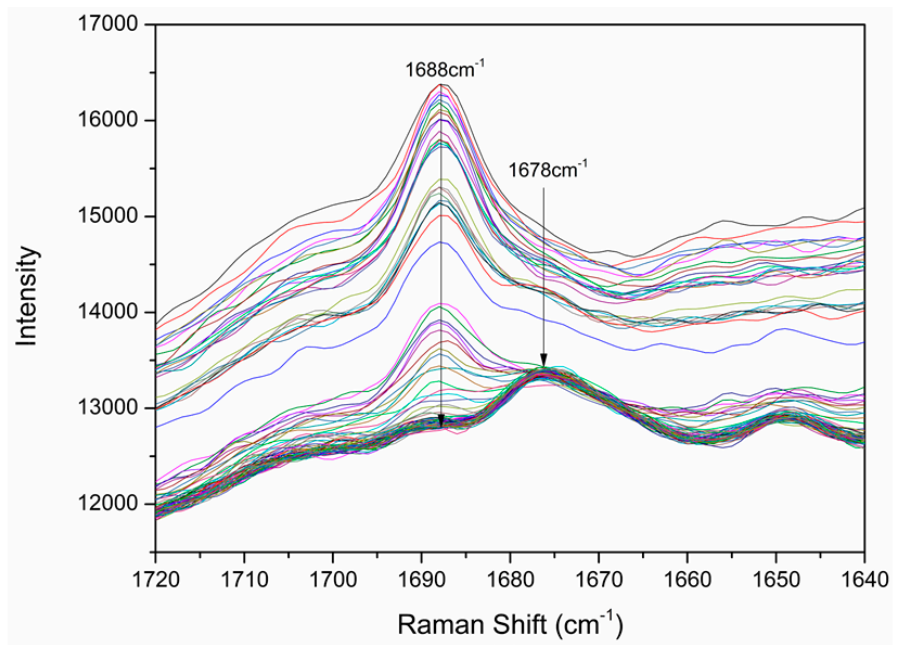

(a)

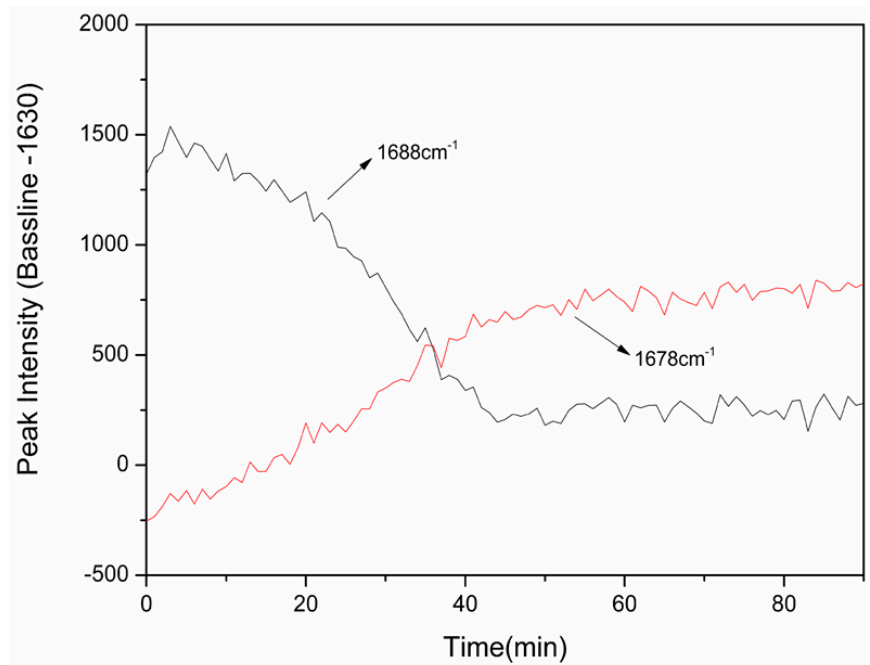

(b)

Figure 5. (a) The variation of Raman spectra at $1640-1720 \mathrm{~cm}^{-1}$; (b) The change of relative peak intensity of $1678 / 1688 \mathrm{~cm}^{-1}$ in TP-BA cocrystal formation in slurry crystallization at $298.15 \mathrm{~K}$. The information about the time interval of the Raman spectra are listed in Table S1.

From the above information obtained from relative intensity change of four couples of characteristics peaks, we know the total time taken for TP-BA cocrystal formation in slurry crystallization under this condition. Therefore, Raman spectroscopy can be used as a reliable and suitable technique to monitor the formation of TP-BA cocrystal and help us to design cocrystallization experiments.

\subsection{Influence of Suspension Density of Raw Materials and Temperature on Theophylline-benzoic Acid Cocrystal} Formation in Slurry Crystallization

To explore the factors affecting TP-BA cocrystal formation process in slurry crystallization, on-line monitoring of the cocrystallization process in solution by Raman spectroscopy was carried out in a methanol/water mixture under different temperatures and suspension densities of raw materials. Besides the above slurry experiment, we added another three sets of experiments at $298.15 \mathrm{~K}$ and $313.15 \mathrm{~K}$ with different suspension densities of raw materials. The corresponding conditions of the four sets of experiments are shown in Table 3. 
Table 3. Temperature, initial concentration, suspension density of raw materials and formation time in TP-BA cocrystal formation process in slurry crystallization.

\begin{tabular}{cccccccc}
\hline & Temperature/K & $\begin{array}{c}\text { Initial } \\
\text { Concentration } \\
\text { of TP/M }\end{array}$ & $\begin{array}{c}\text { Initial } \\
\text { Concentration } \\
\text { of BA/M }\end{array}$ & $\begin{array}{c}\text { Suspension } \\
\text { Density of } \\
\text { TP/M }\end{array}$ & $\begin{array}{c}\text { Suspension } \\
\text { Density of } \\
\text { BA/M }\end{array}$ & $\begin{array}{c}\text { Ratio of TP } \\
\text { and BA in } \\
\text { Cocrystal }^{\text {a }}\end{array}$ & $\begin{array}{c}\text { Formation } \\
\text { Time/min }\end{array}$ \\
\hline $\operatorname{exp~1~}$ & 298.15 & 0.165 & 0.165 & 0.078 & 0.078 & $1: 1$ & 44 \\
$\exp 2$ & 298.15 & 0.130 & 0.130 & 0.042 & 0.042 & $1: 1$ & 57 \\
$\exp 3$ & 313.15 & 0.292 & 0.292 & 0.078 & 0.078 & $1: 1$ & 22 \\
$\exp 4$ & 313.15 & 0.256 & 0.256 & 0.042 & 0.042 & $1: 1$ & 43 \\
\hline
\end{tabular}

${ }^{\text {a }}$ High-performance liquid chromatography (HPLC) chromatograms of product of each set are shown in Supplementary Figure S4.

The peak at $1688 \mathrm{~cm}^{-1}$ was chosen as the characterization peak of raw materials in suspension and the peak at $1678 \mathrm{~cm}^{-1}$ was selected as the characterization peak of cocrystal. The relative peak intensity is presented in Figure 6 (the baseline is the same as above). In Figure 6, the total time taken for cocrystal formation in each experiment under different conditions can be obtained and the formation time was $44 \mathrm{~min}, 57 \mathrm{~min}, 22 \mathrm{~min}$, and $43 \mathrm{~min}$ respectively.

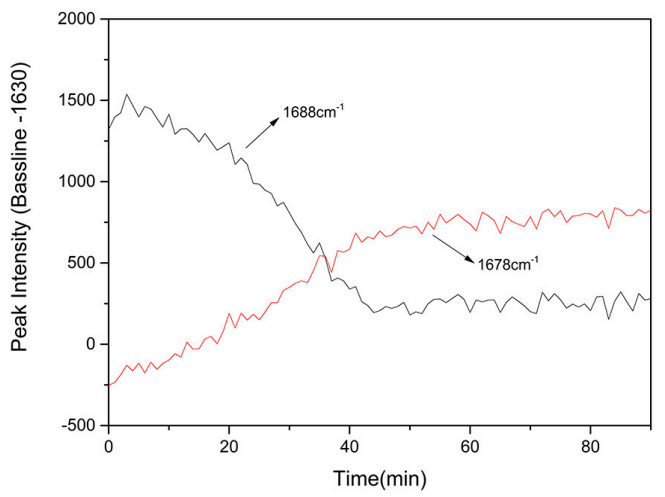

(a)

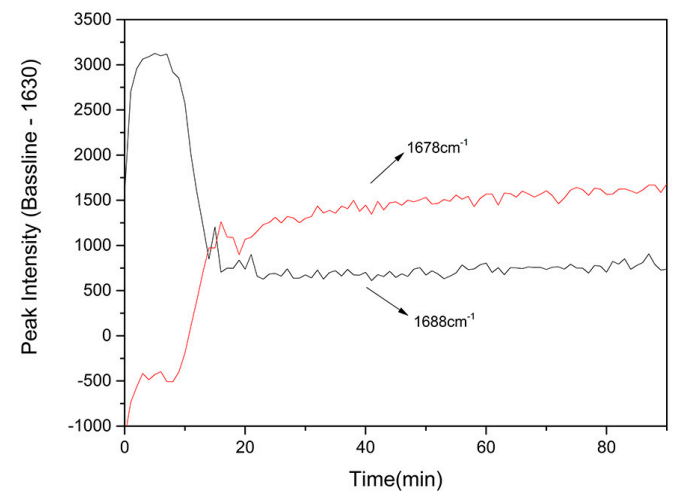

(c)

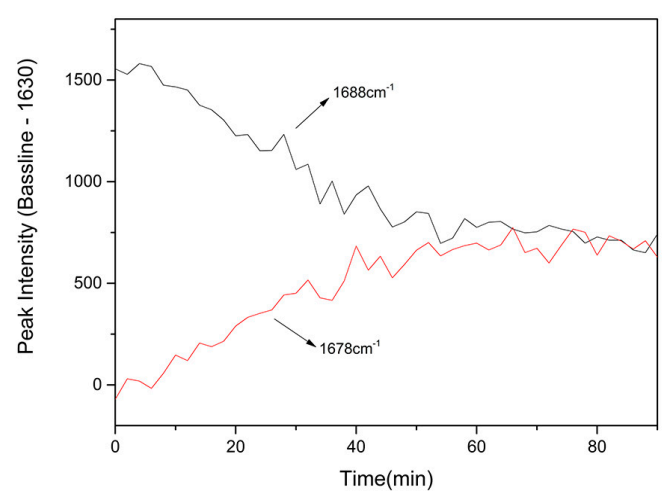

(b)

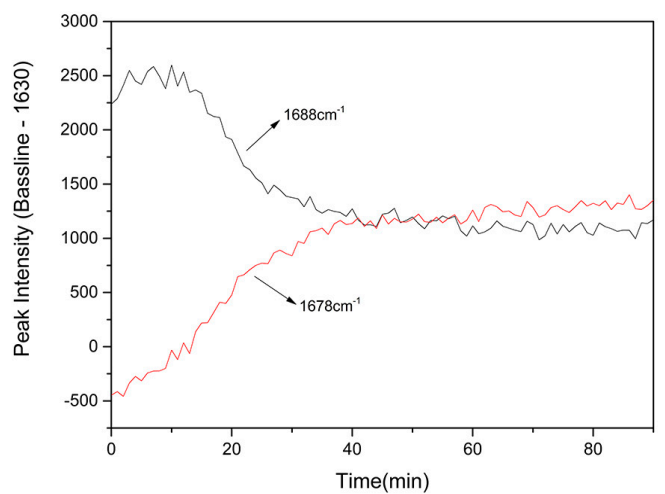

(d)

Figure 6. The relative peak intensity during TP-BA cocrystal formation in slurry crystallization: (a) $\exp 1 ;$ (b) $\exp 2$; (c) $\exp 3$; (d) $\exp 4$.

Comparing the cocrystal formation time under different conditions in slurry crystallization, we found that the suspension density of raw materials and the temperature both have impact on the transformation from raw materials to cocrystal. From the results of exp 1 and 2 (Table 3, Figure 6), the cocrystal formation time of exp 1 is obviously shorter than that of exp 2, while the initial concentration of raw materials in exp 1 is higher than exp 2. The results show that when the initial concentration of raw materials is higher, TP-BA cocrystal formation is faster. The initial concentration directly affects the suspension density of raw materials, and higher suspension density can improve the 
collision probability of raw material particles in slurry crystallization to expedite cocrystal formation. Meanwhile, the total contact surface area between the API and coformer molecules in suspension can be increased as the suspension density becomes higher, and this can also improve cocrystal formation rate. Therefore, the suspension density of raw materials can affect cocrystal formation and the cocrystal formation rate would increase as the suspension density of raw materials increases. The same results can be achieved by comparing the transformation time from raw materials to cocrystal in exp 3 and 4 . The lower formation time in exp 3 indicates higher suspension density in exp $3(0.078 \mathrm{M})$ can achieve a higher rate of transformation from raw materials to TP-BA cocrystal than in exp 4 (suspension density is $0.042 \mathrm{M}$ ) when reaction temperature is same.

Comparing Raman spectra of exp 1 and 3, under the same suspension density condition, TP-BA cocrystal formation is faster in $\exp 3$ when the temperature is higher than that of exp 1 . This is mainly because higher temperature will facilitate the raw materials to reach the activated state with less time, and can thus shorten the formation time. The spectra of on-line monitoring of exp 2 and 4 show the same results. With the same suspension density of raw materials exp $4(313.15 \mathrm{~K})$ has a higher rate of TP-BA cocrystal formation than exp $2(298.15 \mathrm{~K})$, which also indicates temperature has an impact on TP-BA cocrystal formation under the same suspension density condition.

\subsection{On-Line Monitoring of TP-BA Cocrystal Formation Process in Cooling Crystallization}

Similar to the slurry experiments, Raman spectroscopy can be also used to monitor the cooling crystallization process of TP-BA cocrystal. In cooling crystallization, the TP-BA cocrystal was prepared in a methanol/water mixture $(\mathrm{V}: \mathrm{V}=1: 5)$. The Raman spectra obtained from cooling crystallization, which has the same peak shifts as in slurry experiments, can provide various data of the cocrystallization process, including nucleation time, nucleation temperature and suitable cooling ending point.

Two couples of peaks at $1678 / 1688 \mathrm{~cm}^{-1}$ and $918 / 925 \mathrm{~cm}^{-1}$ are regarded as the characteristics peaks to investigate the cocrystal formation process in cooling crystallization. The corresponding relative peak intensity is calculated by the above method and shown in Figure 7. It can be seen from Figure 7 that the peak intensities of 1678 and $925 \mathrm{~cm}^{-1}$, which represent the TP-BA cocrystal, start to increase at $170 \mathrm{~min}$, and those of 1688 and $918 \mathrm{~cm}^{-1}$, which represent the raw materials, begin decreasing at the same time. This means that at $170 \mathrm{~min}$ in the cooling process cocrystal nucleation occurs. Further, the nucleation temperature of TP-BA cocrystal can be calculated from the combined nucleation time and cooling rate, which is at $296.65 \mathrm{~K}$. In theory, during the cooling process the amount of TP-BA cocrystal should keep increasing because the cocrystal solubility decreases continuously as temperature decreases. However, from the Raman spectra of the on-line monitoring of cooling crystallization (Figure 7), the relative peak intensity that represents the cocrystal shows no significant increase and stays at an approximately stable level after $250 \mathrm{~min}$ of the cooling process. That is because when cocrystal solubility decreases to a relatively low level, the yield cannot be increased significantly by further cooling. The corresponding temperature is the ending point of the cooling process. In the TP-BA cooling crystallization process, the ending temperature obtained from the Raman spectra of on-line monitoring is $284.65 \mathrm{~K}$. Hence, on-line monitoring in cooling crystallization by Raman spectroscopy can be used to monitor cocrystal formation and to obtain information about the cocrystal nucleation temperature and ending temperature in cooling crystallization. 


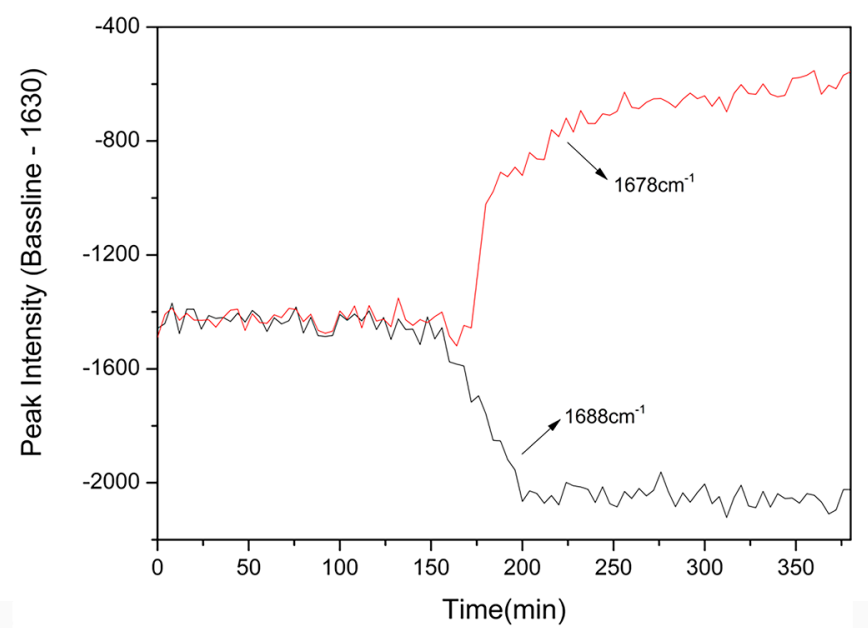

(a)

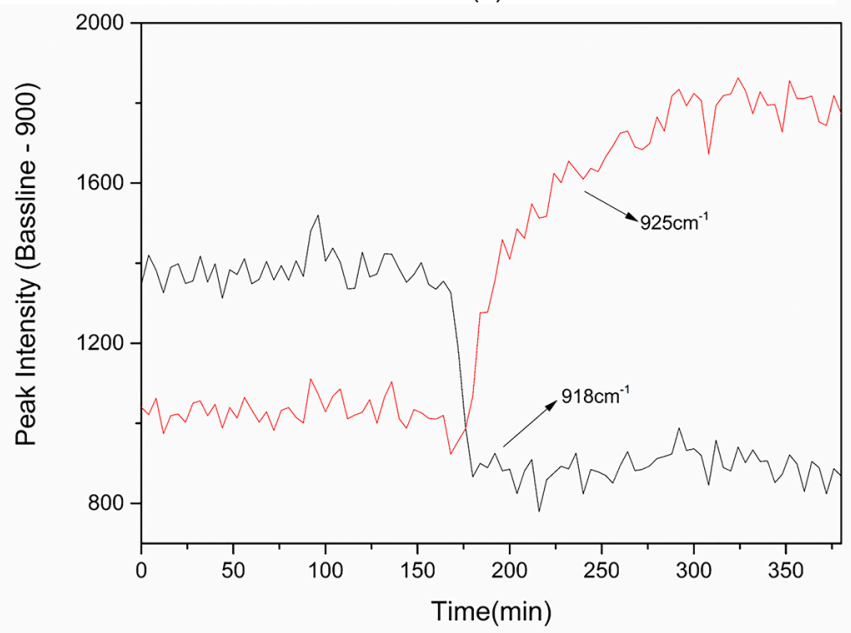

(b)

Figure 7. The relative peak intensity during TP-BA cocrystal formation in cooling crystallization: (a) $1678 / 1688 \mathrm{~cm}^{-1}$; (b) $918 / 925 \mathrm{~cm}^{-1}$.

\section{Conclusions}

In this work, the theophylline-benzoic acid cocrystal was prepared in solution via slurry and cooling crystallization. The solution synthesis of TP-BA cocrystal overcomes the disadvantages of existing preparation methods and can be used to produce large scale and high purity TP-BA cocrystal. The TP-BA cocrystal was characterized by PXRD, DSC, TGA and Raman spectroscopy. The cocrystallization process of TP and BA in solution was monitored by on-line Raman spectroscopy (both slurry and cooling crystallization). The change of peak intensity in the cocrystal formation process can be observed easily from the Raman spectra. The factors affecting the cocrystal formation rate in slurry crystallization were also explored. The results of Raman spectra show that suspension density of drug and coformer, and temperature, both have an impact on TP-BA cocrystal formation. The cocrystallization process will become faster as the suspension density increases. When suspension density of the raw materials is the same, a higher temperature can improve the TP-BA cocrystal formation rate. Temperature and suspension density of raw materials both have a positive correlation with the TP-BA cocrystal formation rate. Furthermore, nucleation temperature and cooling ending point can be obtained by on-line monitoring of the cocrystal formation process in cooling crystallization.

Supplementary Materials: The following are available online at http://www.mdpi.com/2073-4352/9/7/329/s1, Figure S1: (a) The variation of Raman spectra at $1270-1360 \mathrm{~cm}^{-1}$; (b) The change of relative peak intensity of 1323/133 $1 \mathrm{~cm} 1$ in TP BA cocrystal formation in slurry crystallization at 298.15 K. Figure S2: (a) The variation of 
Raman spectra at $1120-1200 \mathrm{~cm}^{-1}$; (b) The change of relative peak intensity of $1161 / 1171 \mathrm{~cm}^{-1}$ in TP-BA cocrystal formation in slurry crystallization at $298.15 \mathrm{~K}$. Figure S3: (a) The variation of Raman spectra at $890-940 \mathrm{~cm}^{-1}$; (b) The change of relative peak intensity of $918 / 925 \mathrm{~cm}^{-1}$ in TP-BA cocrystal formation in slurry crystallization at 298.15 K. Figure S4: HPLC chromatograms of slurry crystallization products in Table 3: exp 1 (a); $\exp 2$ (b); $\exp 3$ (c); $\exp 4$ (d) and cooling crystallization product (e).Table S1: Information about Raman spectra in the range of $1640-1720 \mathrm{~cm}^{-1}$ in Figure 5a. Table S2: Information about Raman spectra in the range of $1270-1360 \mathrm{~cm}^{-1}$ in Figure S1a. Table S3: Information about Raman spectra in the range of $1120-1200 \mathrm{~cm}^{-1}$ in Figure S2a. Table S4: Information about Raman spectra in the range of $890-940 \mathrm{~cm}^{-1}$ in Figure S3a.

Author Contributions: Conceptualization, Y.H., L.Z. and Q.Y.; Formal analysis, W.Y., Y.Y. and C.W.; Funding acquisition, L.Z. and Q.Y.; Investigation, Y.H. and Q.Y.; Methodology, Y.H. and Q.Y.; Project administration, Y.H. and Q.Y.; Supervision, L.Z. and Q.Y.; Writing—original draft, Y.H.; Writing—review \& editing, Y.H., Y.L., Z.Z. and X.Z.

Funding: The support from National Engineering Research Center for Industrial Crystallization Technology (NERCICT) is acknowledged. This work was supported by Major National Scientific Instrument Development Project of China (No. 21527812) and Tianjin Municipal Natural Science Foundation (No. 16JCZDJC32700).

Conflicts of Interest: The authors declare no competing financial interest.

\section{References}

1. Byrn, S.R.; Zografi, G.; Chen, X. Accelerating proof of concept for small molecule drugs using solid-state chemistry. J. Pharm. Sci. 2010, 99, 3665-3675. [CrossRef] [PubMed]

2. Schultheiss, N.; Newman, A. Pharmaceutical cocrystals and their physicochemical properties. Cryst. Growth Des. 2009, 9, 2950-2967. [CrossRef] [PubMed]

3. Aakeröy, C.B.; Salmon, D.J. Building co-crystals with molecular sense and supramolecular sensibility. CrystEngComm 2005, 7, 439-448. [CrossRef]

4. Rodríguez-Hornedo, N. Cocrystals: Molecular design of pharmaceutical materials. Mol. Pharm. 2007, 4, $299-300$. [CrossRef]

5. Liao, X.M.; Gautam, M.; Grill, A.; Zhu, H.J.J. Effect of position isomerism on the formation and physicochemical properties of pharmaceutical co-crystals. J. Pharm. Sci. 2010, 99, 246-254. [CrossRef] [PubMed]

6. Thanigaiman, K.; Khalib, N.C.; Temel, E.; Arshad, S.; Razak, I.A. New supramolecular cocrystal of 2-amino-5-chloropyridine with 3-methylbenzoic acids: Syntheses, structural characterization, hirshfeld surfaces and quantum chemical investigations. J. Mol. Struct. 2015, 1099, 246-256. [CrossRef]

7. Hickey, M.B.; Peterson, M.L.; Scoppettuolo, L.A.; Morrisette, S.L.; Vetter, A.; Guzmán, H.; Remenar, J.F.; Zhang, Z.; Tawa, M.D.; Haley, S.; et al. Performance comparison of a co-crystal of carbamazepine with marketed product. Eur. J. Pharm. Biopharm. 2007, 67, 112-119. [CrossRef]

8. Remenar, J.F.; Perterson, M.L.; Stephens, P.W.; Zhang, Z.; Zimenkov, Y.; Hickey, M.B. Celecoxib: Nicotinamide dissociation: Using excipients to capture the cocrystal's potential. Mol. Pharm. 2007, 4, 386-400. [CrossRef]

9. Zhang, S.; Rasmuson, Å.C. The theophylline-oxalic acid co-crystal system: Solid phases, thermodynamics and crystallization. CrystEngComm 2012, 14, 4644-4655. [CrossRef]

10. Kulla, H.; Greiser, S.; Benemann, S.; Rademann, K.; Emmerling, F. In situ investigation of a self-accelerated cocrystal formation by grinding pyrazinamide with oxalic acid. Molecules 2016, 21, 917. [CrossRef]

11. Basavoju, S.; Boström, D.; Velaga, S.P. Indomethacin-saccharin cocrystal: Design, synthesis and preliminary pharmaceutical characterization. Pharm. Res. 2008, 25, 530-541. [CrossRef]

12. Chieng, N.; Rades, T.; Aaltonen, J. An overview of recent studies on the analysis of pharmaceutical polymorphs. J. Pharm. Biomed. Anal. 2011, 55, 618-644. [CrossRef]

13. Holaň, J.; Štěpánek, F.; Billot, P.; Ridvan, L. The construction, prediction and measurement of co-crystal ternary phase diagrams as a tool for solvent selection. Eur. J. Pharm. Sci. 2014, 63, 124-131. [CrossRef]

14. Ueto, T.; Takata, N.; Muroyama, N.; Nedu, A.; Sasaki, A.; Tanida, S.; Terada, K. Polymorphs and a hydrate of furosemide-nicotinamide 1:1 cocrystal. Cryst. Growth Des. 2012, 12, 485-494. [CrossRef]

15. Friščić, T.; Jones, W. Recent Advances in Understanding the Mechanism of Cocrystal Formation via Grinding. Cryst. Growth Des. 2009, 9, 1621-1637. [CrossRef]

16. Soares, F.L.F.; Carneiro, R.L. Green Synthesis of Ibuprofen-Nicotinamide Cocrystals and In-Line Evaluation by Raman Spectroscopy. Cryst. Growth Des. 2013, 13, 1510-1517. [CrossRef] 
17. Lee, K.S.; Kim, K.J.; Ulrich, J. In Situ Monitoring of Cocrystallization of Salicylic Acid-4,4'-Dipyridyl in Solution Using Raman Spectroscopy. Cryst. Growth Des. 2014, 14, 2893-2899. [CrossRef]

18. Tong, Y.; Zhang, P.; Dang, L.; Wei, H. Monitoring of cocrystallization of ethenzamide saccharin: Insight into kineticprocess by in situ Raman spectroscopy. Chem. Eng. Res. Des. 2016, 109, 249-257. [CrossRef]

19. Kojima, T.; Tsutsumi, S.; Yamamoto, K.; Ikeda, Y.; Moriwaki, T. High-throughput cocrystal slurry screening by use of in situ Raman microscopy and multi-well plate. Int. J. Pharm. 2010, 399, 52-59. [CrossRef]

20. Trask, A.V.; Motherwell, W.D.S.; Jones, W. Physical stability enhancement of theophylline via cocrystallization. Int. J. Pharm. 2006, 320, 114-123. [CrossRef]

21. Abourahma, H.; Urban, J.M.; Morozowich, N.; Chan, B. Examining the robustness of a theophylline cocrystal during grinding with additives. CrystEngComm 2014, 14, 6163-6169. [CrossRef]

22. Alhalaweh, A.; Kaialy, W.; Buckton, G.; Gill, H.; Nokhodchi, A.; Velaga, S.P. Theophylline Cocrystals Prepared by Spray Drying: Physicochemical Properties and Aerosolization Performance. AAPS PharmSciTech. 2013, 14, 265-276. [CrossRef]

23. Fulias, A.; Soica, C.; Ledeti, I.; Vlase, T.; Vlase, G.; Suta, L.M.; Belu, I. Characterization of Pharmaceutical Acetylsalicylic Acid-theophylline Cocrystal Obtained by Slurry Method Under Microwave Irradiation. Rev. Chim. 2014, 65, 1281-1284.

24. Lin, H.L.; Hsu, P.C.; Lin, S.Y. Theophylline-citric acid co-crystals easily induced by DSC-FTIR microspectroscopy or different storage conditions. Asian J. Pharm. Sci. 2013, 8, 18-26. [CrossRef]

25. Lu, J.; Rohani, S. Preparation and Characterization of Theophylline-Nicotinamide Cocrystal. Org. Process Res. Dev. 2009, 13, 1269-1275. [CrossRef]

26. Zhang, S.; Chen, H.; Rasmuson, Å.C. Thermodynamics and crystallization of a theophylline-salicylic acid cocrystal. CrystEngComm 2015, 17, 4125-4135. [CrossRef]

27. Heiden, S.; Tröbs, L.; Wenzel, K.J.; Emmerling, F. Mechanochemical synthesis and structural characterisation of a theophylline-benzoic acid cocrystal (1:1). CrystEngComm 2012, 14, 5128-5129. [CrossRef]

28. Zhang, S.; Rasmuson, Å.C. Thermodynamics and Crystallization of the Theophylline-Glutaric Acid Cocrystal. Cryst. Growth Des. 2013, 13, 1153-1161. [CrossRef]

29. Widhalm, J.R.; Dudareva, N. A familiar ring to it: Biosynthesis of plant benzoic acids. Mol. Plant. 2015, 8, 83-97. [CrossRef]

30. Childs, S.L.; Stahly, G.P.; Park, A. The Salt-Cocrystal Continuum: The Influence of Crystal Structure on Ionization State. Mol. Pharm. 2007, 4, 323-338. [CrossRef]

31. Sheikh, A.Y.; Rahim, S.A.; Hammond, R.B.; Roberts, K.J. Scalable solution cocrystallization: Case of carbamazepine-nicotinamide I. CrystEngComm 2009, 11, 501-509. [CrossRef] 